\title{
TREATMENT OF FIBROIDS.
}

\section{By Professor paul Wernher, of the Rudolf hospital, Vienna.}

\section{(Translated from the German by the Editor.)}

THE former methods of treating myoma of the uterus by galvanization, tincture of ergot, curetting, tamponing of the uterus, \&c., may be considered to be out of date and inapplicable. The results, especially in violent hæmorrhage, are always transitory, and several dangers, such as infection, secondary changes of the myoma, and so on, are inherent to them. To-day only two treatments are worthy of consideration : (I) operative, and (2) treatment by X-ray. If we are consulted by women suffering from fibroma it is our duty first to settle whether the condition is to be treated or not. Severe hæmorrhage, pain, fever, cardiac and stomach troubles are symptoms which point to a necrosis of the fibroma and are indications for treatment. On the other hand, a fibroma causing no symptoms and casually found during examination for other troubles requires no treatment. In such a case it is sufficient to keep the patient under observation and await further developments. The one exception may be when consulted by a woman on account of sterility and in whom a fibroma is found which might possibly be considered the reason for the sterility. In such cases, even though no symptoms of myoma are complained of, an operation may be advisable. The decision whether uterine fibroma should be operated on or irradiated can be arrived at only after very thorough examination and observation of all symptoms by an experienced gynæcologist. Fibroids suitable for irradiation are those in which the only symptom is menorrhagia, all others being better operated on. As the radiation does not have any appreciable influence on the myoma, but only stops the hæmorrhage by destruction of the activities of the ovaries and only later causes shrinking of the fibroma, no women under 45 should be irradiated. As in these cases much more importance is attached to the preservation of ovarian activity, the question of whether very anæmic women should be radiated or operated on is difficult to answer. Personally I prefer in this type of patient the quick operation, viz., vaginal hysterectomy, and in no case am I able to accept the widely-held opinion of using Roentgen rays, because of the great danger of one or two profuse hæmorrhages. Only intra-uterine radium insertion is to be considered in these cases as a lasting and immediate cessation of hæmorrhage. In considering the great importance of a clear division of cases into those where radiation is admissible and those where operation is indicated, a more detailed list of contraindications will be given, which does not mean that in these cases all $\mathrm{X}$-ray treatment must necessarily be useless, but it does mean that it is better replaced by surgical methods. These contra-indications are : (I) uncertainty of diagnosis, specially in fat women, when examination is difficult ; (2) tumours of the ovaries, if very solid and closely connected with the uterus, as they may be mistaken for myomata. If in these cases radiation were to be used, one would go against the general rule that tumours of the ovaries must be operated on. Such tumours may be malignant and during radiation may become inoperable, or else the benign tumours may become malignant-therefore the first condition for radiation is an absolute certainty of diagnosis.

(2) Suspicion of malignancy may be present in two forms: either (a) a combination of myoma and carcinoma in the corpus uteri; or $(b)$ in sarcomatous degeneration of 
fibroma. The combination of $(a)$ is not very rare; both diseases are Suspicion of exceptionally common in nulliparæ and virgins. With careful Malignancy: observation the presence of carcinoma of the hody can hardly be overlooked. Such patients generally complain of irregular hæmorrhage and blood-stained offensive discharge. It is always necessary to do a diagnostic curettage before advising radiation. Sarcomatous degeneration of myoma is very rare, reports vary between 2 per cent. and 5 per cent., but it must be considered in the case of rapid growth of a myoma specially with softening; also in the case of a myoma growing after the menopause. Myomata do not grow after the climacteric, either shrinking or remaining unchanged. Growth without hæmorrhage is always suspicious of malignancy. If some authors state that they have had good results from radiation of sarcomata, I cannot agree with this opinion ; my experience points to sarcoma of the uterus offering an exceptionally bad prognosis for radiation, and I think that the different results reported by authors regarding this condition are due to mistaken diagnosis.

(3) The suspicion of submucous origin. These cases must be operated on because hæmorrhage does not cease after radiation, the hæmorrhage not depending on the ovarian activity but on a purely mechanical origin. It is generally The caused by the rupture of the large thin-walled veins on the surface of

Suspicion of

Submucous Origin. the tumour. Necrosis, caused by insufficient nutrition, may occur after irradiation of submucous fibroids and may render subsequent operation dangerous. The diagnosis of submucous origin is easy, if the myoma hangs in the vagina or at least can be felt after dilatation of the os in the distended cervical canal; but it is our duty, and very often possible, to arrive at a correct diagnosis when the myoma is still hidden in the body and not palpable in the cervical canal.

In these cases many women complain of irregular hæmorrhage and spasmodic pain during the menses. These pains are essentially different from ordinary dysmenorrhœa in that in myoma in the latter the pain generally persists since puberty, while in the former after years of painless menstruation the pain suddenly supervenes. At the examination we find the portio shortened and sometimes almost disappeared. The uterus is often evenly and uniformly enlarged as a submucous myoma is very often single, and of a curious elastic consistency due to the distension of the uterine muscles stretched across the myoma. Careful observation of these symptoms iwill as a general rule enable us to suspect a submucous origin of the myoma, and in such case it is better to avoid radiation.

(4) Such changes are necrosis, cystic degeneration, suppuration, \&c. Secondary If radiation were used in this class of case these dangers and their
Changes. consequences would be increased. Such women do not complain of severe hæmorrhage but of pain, fever, purulent discharge, stomach troubles, \&c.

(5) In judging the importance of symptoms which produce the sensation of pressure one must be very careful. For example, a woman aged 46 complaining of abdominal pressure and frequent micturition may be found, on examination, to

Myoma leading to Pressure on Neighbouring Orsans. have a uterine fibroid the size of a child's fist, the uterus anteverted and mobile. In this case it would be definitely wrong to ascribe the bladder trouble to pressure of the tumour and removal of the uterus would give no improvement in symptoms. A mobile uterus of the size mentioned cannot produce pressure symptoms. This is a case of a very common 
but often unrecognized symptom of the onset of the climacteric and is found in women with normal genitalia. In such a case pressure should not be thought of, even if a tumour of the size mentioned is found, and treatment with an ovarian preparation quickly cures the urinary symptoms. Real pressure symptoms are generally caused in intra-ligamentous or fixed tumours of about the size of a child's head, and whereas the uterus and rectum, owing to their sheltered position, can resist pressure of a tumour for a very long time; the urethra, fixed as it is, does not escape, in consequence women complain of difficulty of micturition. They have to bear down long before micturition occurs, and this difficulty increases during the last few days before menstruation, owing to the onset of hyperæmia and the consequent enlargement of the tumour. If a case such as this is radiated, the symptoms become worse because the radiation of the myoma does not cause shrinkage, but increase in size in the first few weeks and only decreases after two or three months, with the natural result that the patient, finding the symptoms much worse after her treatment by radiation loses patience and prefers to be operated on.

Myoma fixed by Adhesions and

Inflammation of the Adnexa.

(7) Fibroma reaching to the umbilicus should be operated on because in tumours of this size the question arises as to the position of the ovaries and where radiation should be directed to. If the tumour starts from the fundus the ovaries

Fibroma of

Excessive size.

(6) These cases are better operated on. If, after successive radiation the fibroma shrinks, adhesions are pulled apart and that sometimes causes pain. If there is extensive suppuration, the danger is that by traction the pyosalpinx may burst and lead to peritonitis.

may still be confined to the pelvis in spite of the size of the tumour; if the tumour starts from the lower corpus they may be displaced to the? upper surface of the tumour, so it may happen that in spite of the correct dosage being given the ovaries are not subjected to the necessary concentration. Even if the ovaries are properly located and cease their function, it may still happen, that diminution of blood-supply may cause necrosis inside the tumour, which will necessitate surgical operation. This does not imply that in exceptional cases radiation may not be successful in very big tumours. I myself have had a case where a nervous lady with a myomatous uterus reaching far above the umbilicus refused operation and had to be radiated. Complete amenorrhœa ensued, and after the lapse of a year the tumour had contracted in size to that of a child's head, and in later years became even smaller. If you keep to these ideas as to the contra-indications for radiation there still remains about $5^{\circ}$ per cent. of all myoma for radiation. By careful proceeding radiation may give very good results and many failures are avoided. If, on the other hand, you have decided on operative treatment, there remains the question of the type of operation to be done.

\section{There are three different kinds of operation: to be considered.}

(I) Enucleation of the myoma with conservation of the whole uterus.

(2) Supra-vaginal amputation.

(3) Hysterectomy with or without preservation of the ovaries.

Each of the three operations can be performed by abdominal or vaginal methods. Which of these is decided on depends on the judgment of the surgeon, on his training, and on his technique. No doubt in most cases the vaginal operation is more difficult than the abdominal, but for the patient the vaginal methods are less dangerous with quicker convalescence and smaller post-operative complications. 
It is quite certain that thrombosis and embolism which are such drawbacks after operation for myoma are a rarer occurrence with the vaginal than with the abdominal operation. But even a surgeon experienced in vaginal technique would, in cases where it is important to operate as conservatively as possible, and secendly with the view to the possibility of a subsequent pregnancy, favour the abdominal method, even if the size of the tumour and adhesions would not have prevented him from operating by the vaginal method.

Enucleation is specially indicated in solitary myoma of youthful women where children are still desired, with exception of those cases where the tumour is suspended on a pedicle protruding through the os. Even two or three myoma can be successfully removed, but after enucleation of a larger number (unless the tumour is pedunculated) the uterus will be so much damaged that it cannot be considered that it will be able to function again. Again, the convalescence after the operation is generally complicated and grave.

It is always advisable to incise the internal wall of the cervix to get better access to the pedicle. The pedicle should never be severed with scissors, but severed by torsion, as there are often large vessels which might lead to severe hæmorrhage, and be very difficult to deal with. Torsion removes this danger. The cavity of the womb is then .tamponed with gauze which is removed after twenty-four hours. If the subserous or intramural myoma of the body is to be enucleated per vaginam it is advantageous for the surgeon if the myoma is situated in the anterior wall; then it is easy to make the seat of the operation extra-peritoneal by fixing the peritoneum of the bladder to the peritoneum of the uterus. The womb can then be drained per vaginam, but if the tumour is situated on the posterior wall it is better to drain the pouch of Douglas by the posterior fornix.

The alternative to supra-vaginal amputation is total hysterectomy. Some surgeons refuse to consider the supra-vaginal methods because chances of success appear to be threatened by the danger of exudation in the stump, and again because of the possibility of carcinoma supervening. In my opinion the danger of exudation occurring is very remote in correct technique and the occurrence of carcinoma of the stump is very rare. I consider the supra-vaginal method very useful-in the first place it is easy to do especially with unsatisfactory conditions present such as obesity or bad anæsthesia; secondly, because it is possible to preserve a small section of the uterine mucosa of the corpus which enables menstruation to continue for a short time. If the lowest part of the corpus is retained where myoma do not form, the danger of relapse which is sometimes objected to in enucleating operations is almost completely excluded. But of course supra-vaginal amputation is not practical in all cases of myoma. It has indications and contra-indications.

It ought to be performed only if the myoma is fixed to the column as on a stem, and no inflammatory changes of adnexa present. Interligamentary myoma-myoma with pysosalpinx or other inflammatory complications-requires total extirpation, as only by this method can a really effective drainage through the vagina be obtained. Supravaginal amputation with infection of field of operation may easily lead to exudation.

The question whether in panhysterectomy the ovaries should be removed is not decided. Even in young women their function does not persist when left after this operation. In two to three years the menopause gradually supervenes, generally without 
marked disturbance. Again, there is the danger of the formation of cysts and tumours necessitating a second operation. Therefore it is better in women past the climacteric to remove the ovaries per vaginam. Total extirpation of the uterus is not difficult if the uterus is not excessive in size and the vagina yielding.

Radiation can be performed by Roentgen rays or by radium. Consensus of opinion is that the action of radiation is directed to a very small part of the myoma, but mostly to ovaries, which are thus stopped in their action. This means artificial climacteric, as in natural climacteric the hæmorrhage ceases and the myoma recedes after stoppage of hæmorrhage. The exception, perhaps, is the intra-uterine application of radium where the mucous mucosa is destroyed, but sometimes the ovarian activity may persist.

In general, Roentgen rays is the treatment preferred. This treatment can be finished in one sitting or distributed over several treatments in intervals of three weeks. The first method is preferable as its action is quicker. Radiation does not tend to sudden stoppage of hæmorrhage, as in successful radiation one or two or sometimes more menses occur. The hæmorrhage may be more profuse than before, which is important to know if in anæmic women radiation is resorted to. With radiation used immediately after cessation of periods there is generally one more menstruation before lasting amenorrhoea supervenes-when used between periods two to three menses may follow. If the dose is sufficient and the age of patient satisfactory, amenorrhœa is lasting.

Treatment by radium generally done by insertion into the post-fornix or cavitygenerally 30 milligrams used which, adequately filtered, should remain forty-eight hours Generally, intra-vaginal application is sufficient and leads to spontaneous cessation of hæmorrhage, in contrast to using X-rays; it is perfectly harmless but can only be used if myoma at the most fist size. As radium has smaller penetration the ovaries may escape. Its disadvantage also is that fornix may shrink and lead to dyspareunia. This is not present in intra-uterine application, but this method cannot be considered without danger, even with careful application, as septic infection may occur.

These are the principles on which the treatment of patients with myoma should be carried out. If we keep to them we will avoid one-sided preference and unreasonable condemnation of either method and will be able, with each method of treatment, to accomplish the best results. 